\title{
Gehen Sie auf Nummer sicher!
}

- Auch wenn eine topische NSAR-Therapie in der Regel anfänglich vom Arzt veranlasst wird, muss der davon ausgehen, dass der Patient die Behandlung später in Eigenregie fortsetzt. Aus diesem Grund ist es besonders wichtig, von Anfang an ein Präparat zu empfehlen, das problemlos in der Selbstmedikation eingesetzt werden kann.

Bei transdermalen NSAR kommt es vor allem auf gute Hautverträglichkeit und fehlende Toxizität an. So wurde z. B. topischem Ketoprofen von der European Medicines Agency (EMA) kürzlich zwar ein insgesamt positives Nutzen-Risiko-Profil attestiert. Allerdings weist die EMA auch darauf hin, dass das NSAR ein - wenn auch sehr geringes - Risiko für gravierende phototoxische Reaktionen aufweist (1 Fall pro 1 Mio. behandelter Patienten). Die EMA empfiehlt deshalb, topisches Ketoprofen der Rezeptpflicht zu unterstellen und die Warnhinweise bezüglich Sonnenexposition und gleichzeitiger Anwendung des UV-Filters Octocrylen zu verstärken.
Derartige Bedenken bestehen nicht für topische Zubereitungen von Ibuprofen. Unter dem in niedriger Dosierung bereits seit mehr als 20 Jahren rezeptfrei erhältlichen NSAR sind bisher keine phototoxischen Reaktionen beschrieben worden.

\section{Zielgenaue Wirkung, weniger Nebenwirkungen}

Ein seit Kurzem verfügbares Ibuprofen zur transdermalen Applikation ist doc $^{\circledR}$ Ibuprofen Schmerzgel. Sicherheit und Verträglichkeit des Schmerzgels verdanken sich nicht nur dem Wirkstoff, sondern auch der speziellen Galenik. Durch die Verwendung des Hilfsstoffs Dimethylisosorbid liegt Ibuprofen in einer Mikrogelstruktur vollständig gelöst vor, was die Penetration durch die Haut beschleunigt. Daraus ergeben sich mehrere Vorteile:

(1) Die Wirkung tritt sehr schnell, nämlich innerhalb von 15 Minuten ein.

2 Therapeutisch relevante Wirkstoffkonzentrationen werden nur in den Zielgeweben erreicht (z.B. Sehnen, Muskeln,

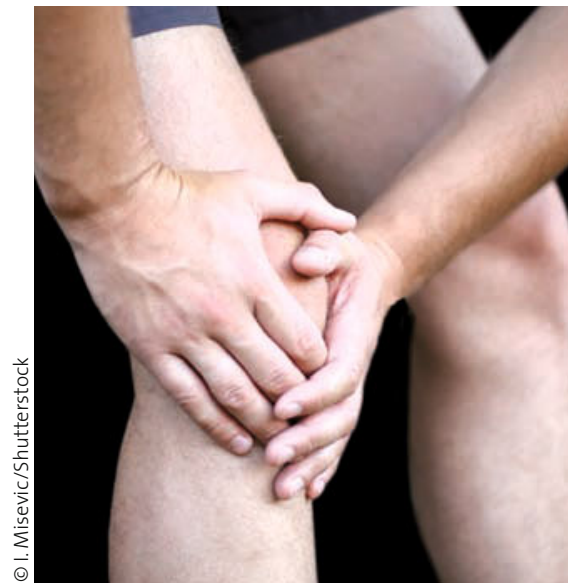

Bei der Bedarfstherapie mit topischen NSAR sind Sicherheit und Verträglichkeit besonders wichtig.

Gelenkkapseln, Synovia). Im Plasma wird die Wirkgrenze $\left(c_{\max } \leq 1 \mu \mathrm{g} / \mathrm{ml}\right)$ nicht überschritten. Dadurch wird das Risiko systemischer Nebenwirkungen minimiert.

(3) Das Mikrogel hinterlässt keine Rückstände auf der Haut.

- Quelle: EMA-Nutzenbewertung von topischem Ketoprofen 22.7.2010; Informationen der Hermes Arzneimittel $\mathrm{GmbH}$

\section{Diabetische Polyneuropathie}

\section{Modernes Antikonvulsivum bessert Schmerz und Schlaf}

- Analgesie allein genügt nicht bei schmerzhafter diabetischer Polyneuropathie. Das Antikonvulsivum Pregabalin wirkt nicht nur schmerzlindernd, sondern verbessert auch die Schlafarchitektur und psychiatrische Komorbiditäten.

Patienten mit schmerzhafter diabetischer Polyneuropathie haben häufig psychiatrische Komorbiditäten. „Etwa jeder Dritte leidet an Depressionen, jeder Vierte an Angststörungen, bis zu zwei Drittel haben Ein- und Durchschlafstörungen“, berichtete Prof. Dr. Göran Hajak, Regensburg. Laut Hajak können sowohl die Schmerzen als auch der daraus resultierende Schlafmangel zur Entstehung psychiatrischer Erkrankungen beitragen.

Deshalb sollten laut Hajak bei der Auswahl eines Analgetikums auch des- sen Auswirkungen auf Schlafarchitektur, Angst und Depression bedacht werden. „Opioide stören den Tiefschlaf und erschweren das Durchschlafen“, gab er zu bedenken. Auch bei den häufig gegen neuropathische Schmerzen eingesetzten Antidepressiva und Antikonvulsiva gebe es Unterschiede: „SSRI stören oft den Schlaf“, so Hajak. „Die dualen Wirkstoffe Duloxetin und Venlafaxin sind hier eher neutral. Nur moderne Antikonvulsiva wirken rundum positiv: Sie sorgen für schnelleres Ein- und besseres Durchschlafen und erhöhen den Tiefschlafanteil.“

\section{Pregabalin ist zweifach wirksam}

Hajak zitierte mehrere, zum Teil noch unveröffentlichte Studien zu dem Antikonvulsivum Pregabalin (Lyrica ${ }^{\circledR}$ ). Demnach verringeren 300 bzw. 600 mg/d Pregabalin die Schmerzen signifikant und erhöhen ebenfalls signifikant die Schlafqualität. „Der verbesserte Schlaf war auch bei Patienten mit Arthrose nachweisbar, bei denen Pregabalin keinen Einfluss auf den Schmerz hatte", betonte Hajak. In anderen Studien hatte Pregabalin Tiefschlaf induziert bzw. generalisierte Angst hochsignifikant verringert. „Um eine diabetische Polyneuropathie und komorbide psychiatrische Störungen zu therapieren, genügen meist moderne dual wirkende Antidepressiva und dazu ein Antikonvulsivum wie Pregabalin“, so Hajaks Fazit.

- Simone Reisdorf Symposium, Jahrestagung der Deutschen Diabetes-Gesellschaft, Stuttgart, 12. Mai 2010 (Veranstalter: Pfizer) 